\title{
A Combining Rate Adaptation Scheme of Channel Coding and Network Coding in None-relay Downlink Wireless System
}

\author{
ZiJun Shi ${ }^{\mathrm{a},{ }^{*}}$, ZhiPing $\mathrm{Shi}^{\mathrm{a}}{ }^{\text {,*2 }}, \mathrm{Gu} \mathrm{Li}^{\mathrm{a}}$ \\ ${ }^{a}$ National Key Laboratory of Communication, University of Electronic Science and Technology of China, \\ Chengdu, China
}

\begin{abstract}
We propose rate adaptation in the entire downlink process of transmission in channel-network combining coding systems. In current literatures, rate adaptation is discussed in channel coding or network coding separately and in traditional channel-network combining coding cases, network coding rates are generally 1. This paper is based on a one-source multi-destination wireless downlink communication system and a novel scheme of rate adaptation is applied. Our scheme of adaptation works between channel coding rate and network coding rate and its influence on system performance, which is given in form of simulation in the last chapter of this paper, shows that it works well for deducing system BER.
\end{abstract}

Index Terms: Network Coding; Rate Adaptation; Combining Coding Rate

(C) 2012 Published by MECS Publisher. Selection and/or peer review under responsibility of the Research Association of Modern Education and Computer Science

\section{Introduction}

Since network coding is proposed by Ahlswedeet al. [1] in 2000, it has attracted much attention and been researched a lot. As the broadcasting property of wireless communication suits wireless cooperation scheme well, network coding is performed on physical layer, improving system's noise immunity while obtaining larger system throughput in form of network coding.

Recent approaches on rate adaptation of network coding are mostly focused on rate adaptation on network coding alone [2][3], showing us the benefits that can be achieved by rate-adapted network codes. This paper shows that the rate adaptation between network coding and channel coding can also affect the performance of the entire transmission system.

In the second part of this paper we describe the system model we use in our analysis which is similar to the practical communication system, and in part III, we provide the channel-network coding rate adaptation scheme. In part IV, a simulation result and its corresponding analysis is given. The last part of this paper lists out all the papers we referred to during the research.

Supported by Youth Foundation of University of Electronic Science and Technology of China and National 973 project (2007cb310604)

* Corresponding author:

E-mail address: ${ }^{* 1}$ juicyshizi@163.com; ${ }^{* 2}$ szp@uestc.edu.cn 


\section{A Downlink Wireless System Model}

The wireless downlink transmission system we use contains one source which acts as base station and 4 receivers which act as users. Each user can perform as a relay for other users in a sequence which is decided according to the source-receiver channel condition of each receiver node. System model is shown in Fig.1, similar to the system model proposed in reference [4].

In this model, the source node is to transmit a sequence of bits to all the receiver nodes. It firstly channel encodes the data and broadcasts them, then the receivers decode the information they hear separately, decide in what way a receiver should network encode its decoded data and figure up how much time it should wait to broadcast as a relay. For each receiver, both the network encoding method and the waiting time are determined by the transmission quality of his own source-receiver channel [4].

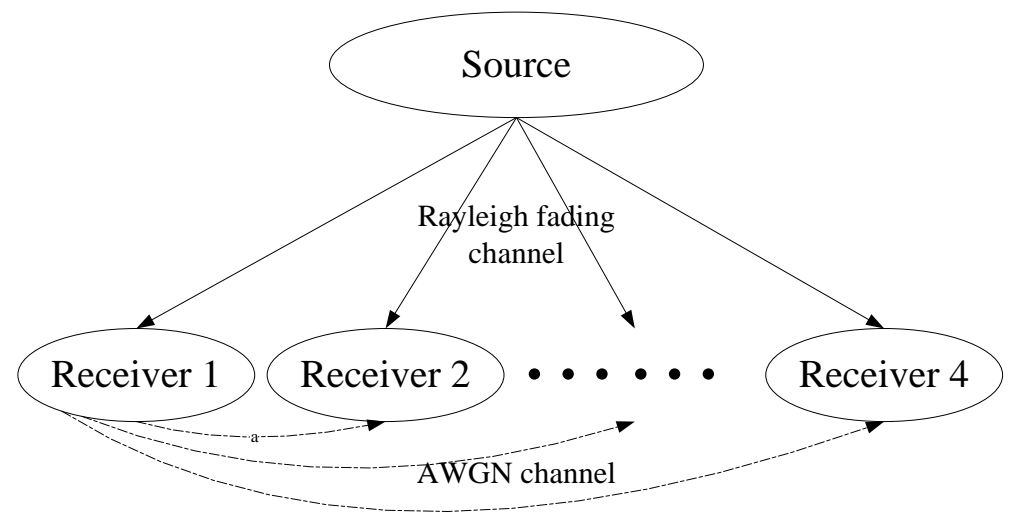

Fig. 1. Wireless downlink system model

Phisan Kaewprapha put forward a very clever scheme of channel-network coding combination [4].This scheme is given in a one-source multi-receiver system. We assume that there $\operatorname{are}^{m}$ receivers in the system, each packet that the source broadcasts is of length $L$ and the source is to transmit $k \times m \times L$ bits to the receivers where $^{k}$ is a positive integer. We also assume that the network coding matrix is called $A$.

Channel-network combining decoding matrix

\begin{tabular}{l|l|l|l|l|l|l|l|}
\hline$\pi_{1}$ & $\pi_{2}$ & & $\pi_{3}$ & $\mathrm{e}$ & & & \\
\hline & $\pi_{4}$ & $\pi_{5}$ & & & $\mathrm{e}$ & & \\
\hline$\pi_{6}$ & & & $\pi_{7}$ & & & $\mathrm{e}$ & \\
\hline & & $\pi_{8}$ & $\pi_{9}$ & & & & $\mathrm{e}$ \\
\hline
\end{tabular}
network coding
matrix $\mathrm{A}$

Fig. 2. An example of network coding matrix and channel-network combining decoding matrix 
In this scheme, each chunk $\left(\pi_{\mathrm{i}}\right)$ of $A$ is a $L \times L$ quasi-cyclic unit matrix and none of the chunks are the same. Network coded bits are obtained by multiplying relay's channel decoded bits with network coding matrix $A$. Each node does such a multiplication and broadcasts its network encoded bits by turn.

The ultimate combining decoding matrix is a concatenation of $A$ and a $(L \times \mathrm{m}) \times(L \times \mathrm{m})$ unit matrix or a lower triangular matrix, and this matrix as a whole is deemed as a QC-LDPC decoding matrix, whose input decoding information is a concatenation of a certain node's channel decoded bits and all of its received network encoded bits from other receiver nodes.

\section{Calculation of Systematic Coding Rate}

System coding rate $(R)$ is defined as ratio of the number of bits to be transmitted to the receivers $(K)$ and the number of bits that the whole system actually transmits $\left(N^{N}\right)$.

$$
R=\frac{K}{N}
$$

We assume in the transmission process that when source broadcasts, it encodes the bits previously at the rate of $R_{1}$ and receivers separately channel decode their received information, after that, relay network encodes its decoded information at network coding rate ${ }^{R_{2}}$. Calculating method of system coding rate is shown in detail in Fig.3.

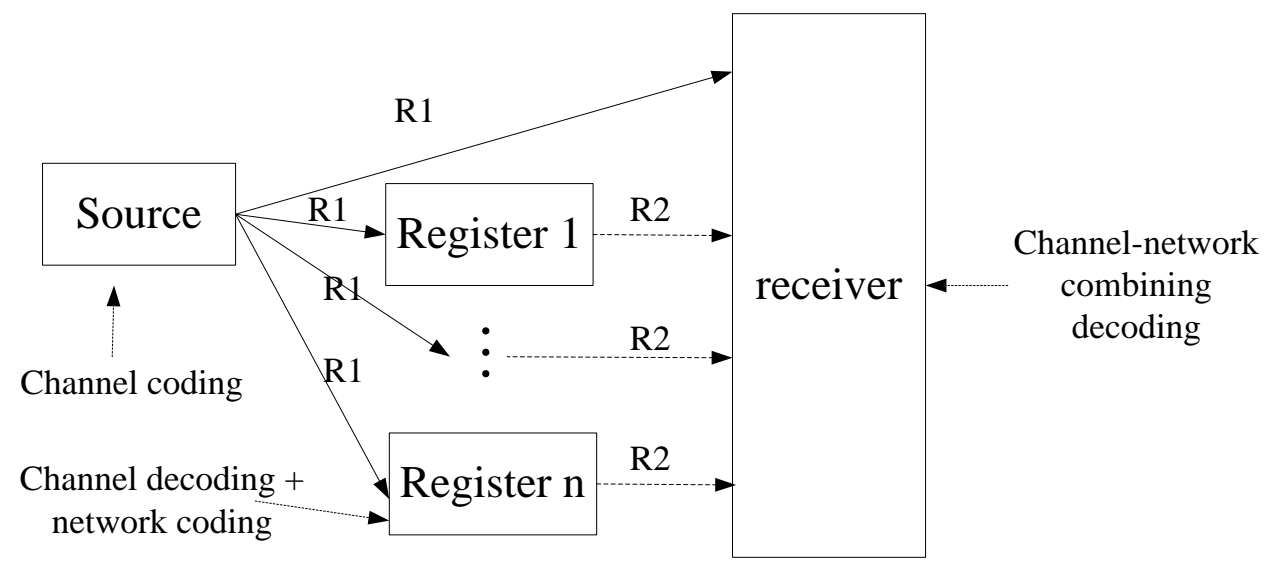

Fig. 3. Calculation for system coding rate

In Fig. 3 we assume that the source intends to transmit ${ }^{W}$ bits of information to the receivers, it firstly channel encodes the $W$ bits into $W / R_{1}$ bits and then broadcasts them. Receivers channel decode the ${ }^{W / R_{1}}$ bits into $W$ bits and the one or many receivers who act as relay network encodes its $W$ bits into $m \times W / R_{2}$ bits and broadcasts them. As a result, the number of bits the system totally broadcasts is $W / R_{1}+m \times W / R_{2}$. Thus, we get the system coding rate: 


$$
R=\frac{W}{W / R_{1}+m \times W / R_{2}}
$$

What is worth to mention is that as all bits are transmitted with the same amount of power, the whole system uses the same amount of power in different process of transmission as soon as each time it has a same system coding rate, providing a fair platform for the rate adaptation between ${ }^{R_{1}}$ and $R_{2}$

\section{Combining Rate Adaptation Scheme}

\section{1. network coding using a whole matrix}

In this scheme of network coding, we firstly adapt the network coding matrix(called $A$ ) according to network coding rate and then the receiver acting as relay multiply its channel decoded information by the whole network coding matrix to get the network coded bits, which are later broadcasted to other receivers.

Let:

We assume the size of $A$ is $M \times N$.

$$
M=L \times \mathrm{m} / R_{2} ; N=L \times m
$$

Thus, after multiplying the receiver's decoded bits by $A$, we get $m / R_{2}$ column vectors of length $L$, all of which is connected into a $\left(L \times \mathrm{m} / R_{2}\right) \times 1$ column vector, which is deemed as the network coded information.

The ultimate combining decoding matrix is a concatenation of $A$ and a $\left(L \times \mathrm{m} / R_{2}\right) \times\left(L \times \mathrm{m} / R_{2}\right)$ unit matrix or a lower triangular matrix, one example of such network coding and channel-network combining decoding matrix is given in Fig.4.

\section{channel-network combining decoding matrix}

\begin{tabular}{|l|l|l|l|l|l|l|l|l|l|}
\hline$\pi_{1}$ & & $\pi_{2}$ & & $\mathrm{e}$ & & & & & \\
\hline & $\pi_{3}$ & & $\pi_{4}$ & & $\mathrm{e}$ & & & & \\
\hline$\pi_{5}$ & $\pi_{6}$ & & & & & $\mathrm{e}$ & & & \\
\hline$\pi_{7}$ & & & $\pi_{8}$ & & & & $\mathrm{e}$ & & \\
\hline & $\pi_{9}$ & $\pi_{10}$ & & & & & & $\mathrm{e}$ & \\
\hline & & $\pi_{11}$ & $\pi_{12}$ & & & & & & $\mathrm{e}$ \\
\hline
\end{tabular}
network coding
matrix A

Fig. 4. An example of network coding matrix when network coding with a whole matrix 


\section{2. network coding line by line}

In this scheme of network coding, after adapting the size of the network coding matrix $A$, we correspondingly add a lower triangular matrix to it, obtaining the final channel-network combining decoding matrix (called $B$ ), which is used in both the network coding phase and the channel-network combining decoding phase. When network coding, we use each line of matrix $B$ to network encode the data a relay gets from the source node. An example of such channel-network coding matrix is shown in Fig.5.

\section{Matrix used for both network coding and channel-network combining decoding}

\begin{tabular}{|c|c|c|c|c|c|c|c|c|c|}
\hline$\left(\pi_{1}\right.$ & & $\pi_{2}$ & ) & e & & & & & \\
\hline( & $\pi_{3}$ & & $\pi_{4}$ & ) & e & & & & \\
\hline$\left(\pi_{5}\right.$ & $\pi_{6}$ & & & $\pi_{13}$ & ) & e & & & \\
\hline$\left(\pi_{7}\right.$ & & & $\pi_{8}$ & & $\pi_{14}$ & $\left.\pi_{15}\right)$ & e & & \\
\hline ( & $\pi_{9}$ & $\pi_{10}$ & & $\pi_{16}$ & & & $\pi_{17}$ & e & \\
\hline ( & & $\pi_{11}$ & $\pi_{12}$ & & $\pi_{18}$ & & & $\pi_{19)}$ & e \\
\hline
\end{tabular}

Fig. 5. An example of network coding matrix when network coding line by line

The first relay, correspondingly the best receiver, multiplies its decoded data (a $m \times L \times 1$ column vector) with the bracketed part in the first line of matrix $B$, gets its own network coded bits and transfers them to the other receivers. The second relay firstly combines its own channel decoded data with the network coded data from its previous relay into a $(m+1) \times L \times 1$ column vector, then multiplies it with the bracketed part of the second line of matrix $B$, and finally broadcasts its $L \times 1$ network coded column vector to other nodes. All of the other nodes act the same way separately.

There are two cases in this scheme:

When line number of $B$ is larger than the number of receiver nodes, we recommend earlier relays (who have better source-receiver channels) to network encode more times than the latter ones.

When the number of lines of $B$ is less than that of receiver nodes, we recommend only the first several relays perform network encoding.

After all the relays finish network coding and broadcasting, each of the nodes combines its own decoded data from the source and all the network coded data both generated by itself and received from other receivers together, which is used finally to perform channel-network combining decoding using matrix $B$. 


\section{Simmulation and Result Analysis}

Our simulation model includes a remote source node and 4 receivers who can also act as relays. In order to meet the practical applicability, we assume that the channels between source and receivers are Rayleigh fading and channels between receivers are AWGN channels. As the source-receiver channels are inhomogeneous, the Rayleigh fading channels SNRs are arranged to randomly distribute within $[(\gamma-3),(\gamma+3)]_{\mathrm{dB}}$, and the SNR value between receivers in AWGN channels is $\gamma$. We use LDPC codes for channel coding and when perform network encoding, we use the line-by-line network coding method. Simulation results are shown in Fig.6

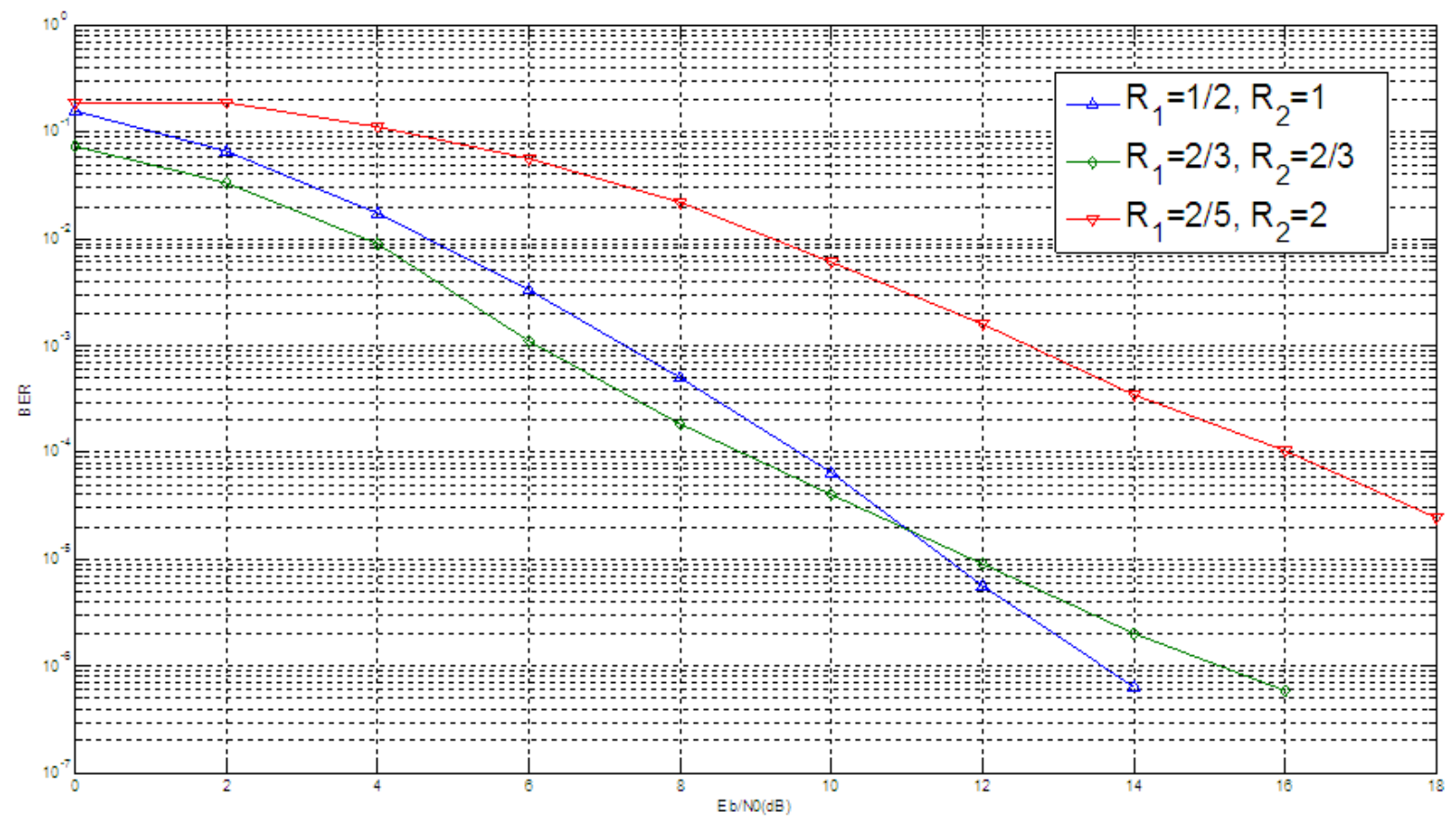

Fig. 6. Simulation result for 3 different rate pairs

As is shown in Fig.6, in order to make sure that in all cases we have the same system coding rate, we need to decrease the network coding rate $R_{2}$ as the channel coding rate $R_{1}$ increases. We can see in the figure that as channel coding rate decreases the system transmission performance gets better. The reason lies in the fact that the source-receiver channel is worse than the receiver-receiver AWGN channel, which needs more check bits to guarantee the transmission quality. But as the network coding rate gets too large, the system performance gets worse because in this case, the network coded information which act as the check bits for the final channel-network combining decoding is far less than enough. Available rate pairs are abundant and as the number of bits to be transmitted gets larger, the number of available rate pairs gets more and more.

\section{Conclusion}

In this paper, we perform rate adaptation between channel coding and network coding in wireless downlink transmission systems. Two schemes for adapting network coding rate are given. At the end of the paper, 
simulation result on channel-network rate adaptation is given and the influence of it on system performance is obvious.

We found that as the source-receiver channel is much worse than the receiver-receiver channel in practice, it's wise, to some distance, to decrease the channel encoding rate $R_{1}$ to get a better system performance. But as the value of $R_{1}$ gets too small, the network coding rate needs to turn rather large to achieve the same system coding rate, in which case the system performance can turn bad as a result of the lack of network coded information which can work as the check bits for the final channel-network combining decoding.

\section{References}

[1] R. Ahlswede, N. Cai, S. Y R. Li, and R. W. Yeung, "Network infonnation flow," IEEE Trans. Infomation Theory, vol. 46, no. 4, pp. 1204-1216, 2000)

[2] Zihuai Lin, Branka Vucetic," Power and Rate Adaptation for Wireless Network Coding with Opportunistic Scheduling", ISIT 2008, Toronto, Canada, July 6 - 11, 2008

[3] Yuchul Kim and Gustavo de Veciana," Is Rate Adation Benieficial For Inter-Session Network Coding?"

[4] Phisan Kaewprapha, Nattakan Puttarak, Jing Li (Tiffany), Zhiping Shi," Cooperative Data Dissemination Exploiting Offset Random Network Coding”, Journal of Applied Remote Sensing(submitted) 